\title{
UNIVERSITYOF
}

FORWARD

THINKING

WESTMINSTER用

WestminsterResearch

http://www.westminster.ac.uk/westminsterresearch

Intra-acting with the IceCube Neutrino Observatory, or; how the technosphere may come to matter

Thomson, J. and Engelmann, S.

This is a copy of the accepted author manuscript of the following article: Thomson, J. and Engelmann, S. (2017) Intra-acting with the IceCube Neutrino Observatory, or; how the technosphere may come to matter. Anthropocene Review, 4 (2), pp. 81-91. The final definitive version is available from the publisher Sage at:

https://dx.doi.org/10.1177/2053019616683726

(C) The Author(s) 2017

The WestminsterResearch online digital archive at the University of Westminster aims to make the research output of the University available to a wider audience. Copyright and Moral Rights remain with the authors and/or copyright owners.

Whilst further distribution of specific materials from within this archive is forbidden, you may freely distribute the URL of WestminsterResearch: ((http://westminsterresearch.wmin.ac.uk/).

In case of abuse or copyright appearing without permission e-mail repository@westminster.ac.uk 
Intra-acting with the IceCube Neutrino Observatory; or, how the technosphere may come to matter

\begin{abstract}
This paper contends that a robust concept of the technosphere - indeed one that is truly adequate to the Anthropocene - must be approached using a plurality of methods that do not categorize agencies or rely on hierarchical scalar analysis. In this commentary, we draw from feminist science studies scholar Karen Barad's philosophy of agential realism, and in particular her concept of "intra-action," to identify the technosphere as emergent from entangled practices, sites and infrastructures, and to trace the technosphere from the "meso" scale to subatomic and cosmological realms of force and energy. We demonstrate the value of a critical, intra-active approach to technical assemblages by thinking the technosphere concept with and within a vast experimental apparatus: the IceCube Neutrino Observatory.
\end{abstract}

\title{
Keywords
}

intra-action, neutrino, agency, scale, entanglement, technosphere, cascade, IceCube 
In my agential realist account, humans do not merely assemble different apparatuses for satisfying particular knowledge projects; humans are a part of the configuration or ongoing reconfiguration of the world - that is, they/we too are phenomenon. In other words, humans (like other parts of nature) are of the world, not in the world, and surely not outside of it looking in. (Barad, 2007; 206)

Introduction

The concept of the 'technosphere' as articulated by Peter K. Haff suggests that technical objects, institutions, and processes have coalesced into a novel planetary sphere to which humans, and indeed other living and non-living things, are 'subservient parts' in this sphere's search for 'quality energy' (Haff, 2014). The technosphere concept therefore embodies a radical redrawing of what constitutes the technical in the age of the Anthropocene, and for this it is of great value. In this commentary, however, we articulate a few reservations regarding the methods and frameworks of research that are enrolled and enabled by the technosphere. In particular, we are critical of Haff's granting of hierarchical autonomy to the technological systems that are embedded in the material, discursive and energetic flows across Earth. We are critical of this gesture not for some nostalgic ideas about free will, or romantic concepts of Nature. Rather, the technosphere's reliance on hierarchical concepts of agency and scale limits critique itself, foreclosing our capacities to witness the diverse species of onto-, epistemo-, and techno-logics emerging on our planet.

To apprehend the 'becoming-technology of the world' announced in the technosphere, our capacities to think, act and invent must become adequate to what Haff (2014) refers to as technologies' 'escaped' velocities. In this endeavor, we can gain valuable insights from literature in science studies and posthumanist philosophy that radically extends notions of accountability, agency and causality (Bennet, 2010; Braidotti, 2013; Pickering, 1994). Specifically, through an engagement with physicist and 
philosopher Karen Barad's (2007) critical practice of agential realism, and especially her concept of intra-action, we address several questions important to a meaningful articulation of Earth's technical capacities. Moreover, by exploring intra-action through a site - the IceCube Neutrino Observatory - we test spatial concepts that transcend scales, and depart from the scale-based 'graining' of a 'techno-sphere'. In the Anthropocene, the stakes for such new concepts are high. Before engaging further with the technosphere concept we briefly set the critical-conceptual stage for our contribution. While doing so, we define the Baradian terms of agential realism and intra-action and delineate their consequences for (re)animating agency and scale.

New knowing subjects

Accompanying the figure of an Onomatophoric Anthropocene (Szerszynski, 2014) are challenges to human exceptionalism. These challenges emerge from the envelopment and exposure of bodies to the 'volatile juncture' (Massumi, 2002) of hybrid and toxic Anthropocene ecologies. Recent interdisciplinary work across science studies, geo-philosophies, posthumanism, and cybernetics has suggested: 'This new knowing [Anthropocenic] subject is a complex assemblage of human and non-human, planetary and cosmic, given and manufactured, which requires major re-adjustments in our ways of thinking' (Braidotti, 2013: 159; emphasis ours). Such 're-adjustments' are found in Karen Barad's agential realism, which depends on a performative, as opposed to representational, understanding of phenomena. Barad inherits her approach from scientist and philosopher Donna Haraway's 'diffractive methodology'. To employ the optical metaphor of diffraction is to step beyond representation as a stance that presumes critical displacement between subject and world (Haraway, 1997). Barad's critical method, then, "is a way of understanding the world from within and as a part of it" (Barad 2007: 88). Agential realism is the practice of attuning to differences, frequencies, interferences and entanglements of intra-acting entities, 
apparatuses, theories and agencies. As she further underlines, 'Knowing does not come from standing at a distance and representing, but from a direct material engagement with the world' (Barad, 2007: 49).

The neologism intra-action plays a significant role in Barad's 'relational ontology' as well as in this paper. Like Andrew Pickering's (1994) ‘dance of agency', Barad's (2007) intra-action may be understood as, "the mutual constitution of entangled agencies" (Barad, 2007: 33). In other terms, intraaction's contribution is that agency does not belong to discrete entities but rather emerges from the ongoing processes of relations themselves. In Barad's social constructivist approach, and in other posthumanist accounts, agency, 'is cut loose from its traditional humanist orbit' (Barad, 2007: 235). 'Crucially,' Barad emphasizes, 'agency is a matter of intra-acting; it is an enactment, not something that someone or something has' (Barad, 2007: 235). Going deeper, she explains intra-action's position vis-àvis complex systems theory:

...intra-actions effect the rich topology of connective causal relations that are iteratively performed and reconfigured. This is a reworking of causality that goes beyond not only its classical conception but also goes beyond that of complex systems theory as well: "emergence," in an agential realist account, is dependent not merely on the nonlinearity of relations but on their intra-active nature (i.e., on nonseparability and nontrivial topological dynamics as well). Barad, 2007: 393

In this account of intra-action we find the radical proposal that all forms of matter, even subatomic particles like quarks and leptons, together with the mathematical theories and equations used to describe them, are in a flux of being 'made and unmade' with many other entities across scales and with other compositions (Barad, 2007). It is important to note that what is 'made and unmade' are the boundaries that come to define 'subjects' and 'objects,' as well as the boundaries that categorize and divide different scales of analysis. What constitutes a limit, a sphere, or a 'sub-' anything is constantly up for negotiation. Distinctions are never fixed, but are dependent on sensing apparatuses, theories, forms of mathematical 
description, modes of observation, or other investigative cultural practices, which in turn rely on economic, social and ecological factors.

This brings us to the provocation of this paper: abstracting the technical 'sphere' away from the material, the creaturely and the cosmological neglects the insight that Earth's technical capacity is an intra-acting space of entangled agencies. Phrased differently, technological apparatuses and infrastructures are not autonomous, but co-constituted through social, economic and bio-geo-physical processes. This provocation implies the following question: what forms of analysis of technology might be opened up if we unsettle fixed notions of agency and scale, probing the, 'rich topology of connective causal relations' (Barad 2007, 393) that transcend the liminal boundaries of our planet's 'spheres'? In addressing this question, the neutrino becomes a meaningful collaborator. This paper will continue to demonstrate the value of an intra-active approach to technical assemblages by (re)thinking the technosphere concept with a vast, experimental apparatus: the IceCube Neutrino Observatory.

\section{IceCube Neutrino Observatory}

We had no choice, nature had built the detector for us. - Dr. Francis Halzen, director of the IceCube Observatory, personal interview with authors, 2015.

The IceCube Neutrino Observatory, located at the geographic South Pole, represents one of the largest, strangest, and most complex collaborations in the history of scientific research. Its utility for thinking through Haff's technosphere concept became evident in the process of a multi-sited ethnography through which many voices (human and nonhuman) were heard and echoed. Over the past two years, we analyzed recent publications emerging from the IceCube Neutrino observatory, contacted members of the core team of researchers there, and interviewed the current director of IceCube, Dr. Francis Halzen. We also interviewed and visited the laboratory of Dr. Christian Spiering, former director of the Baikal 
Neutrino Telescope and IceCube spokesperson between 2005 and 2007. We touched Digital Optical Modules - the 'eyes' of the IceCube Observatory - and watched the gorgeous signals of neutrinos cascading in ice with $\mathrm{PhD}$ and Postdoctoral students. We spent one week at the Cryogenic Underground Observatory of Rare Events (CUORE) at the Laboratory del Gran Sasso in Italy, where a number of experiments aim to understand neutrino oscillation and decay. ${ }^{1}$ Among many others, these encounters helped us to accurately attend to neutrino physics, and have informed what we understand to be the notable contributions of IceCube to the field(s) of particle physics, astronomy and cosmology, as well as to notions of Earth's technical capacities.

The omnipresent yet phantom-like neutrino, of which trillions are passing through this page at any instant, summons an entirely new genre of experimental physical observation, and is speculated to be integral to our future understanding of non-baryonic or "dark" matter and energy - the stuff that comprises 96 percent of the cosmos. Unlike other particles, neutrinos interact only via the 'weak force': the force that governs particle decay, and that which is fundamental to fusion and fission processes. In other words, most other particles have charge and significant mass, making them susceptible to electromagnetic fields and therefore determination, experiment, observation, measurement, and manipulation. In contrast, neutrinos are not affected by electromagnetism, and they find baryonic matter completely porous: it has been calculated that a neutrino could pass through 100 Light Years of lead without slowing down. Thus, while neutrinos have been often called "the ideal cosmic messengers" (Halzen, interview with authors, 2015) approaching Earth from the far reaches of the universe, they are nevertheless imperceptible. We can only sense neutrinos as traces, when they very rarely decay in charged matter, producing electromagnetic and hadronic showers, or cascades. For the purposes of this paper, a cascade is a shower of secondary charged particles from the event of a high-energy neutrino

\footnotetext{
${ }^{1}$ This paper emerges out of a multi-sited para-ethnography (Holmes and Marcus, 2006) that is not limited to academic work; it has also inspired artworks made in collaboration with neutrino sensing experiments. In particular, our visit to the Cryogenic Underground Observatory for Rare Events (CUORE) where physicists study 'neutrinoless double beta decay' was part of the research phase for a video art project produced by one of this paper's authors.
} 
intra-acting with matter. The quality and orientation of the cascade's shower of secondary particles holds layers of information that pertain to the neutrino's characteristics and journey.

Neutrino detection requires a clearing of the field. Most 'neutrino telescopes' are situated deep underground in repurposed mines or purpose-built caverns; for example, the CUORE experiment visited by this paper's authors is situated $1.4 \mathrm{~km}$ under the Abruzzo Mountains in Italy. In the case of detectors such as the Super-Kamiokande detector in Japan, a 50.000 litre tank of ultra-pure water buried deep within the Japanese Alps, the rock above the sensor apparatus acts as a shield from other high energy particles like cosmic rays that shower the planet. One of the youngest and most prolific of these telescopes, and that which has been the subject of our research - IceCube - is embedded at depths between 1450 and 2450 metres within the 200,000 year old Antarctic ice shelf at the Amundsen-Scott South Pole Station. Some of the experiments at IceCube in fact capture neutrinos that originate from the other hemisphere of the earth. These neutrinos pass through the whole planet and are detected in the IceCube apparatus moving 'upwards'.

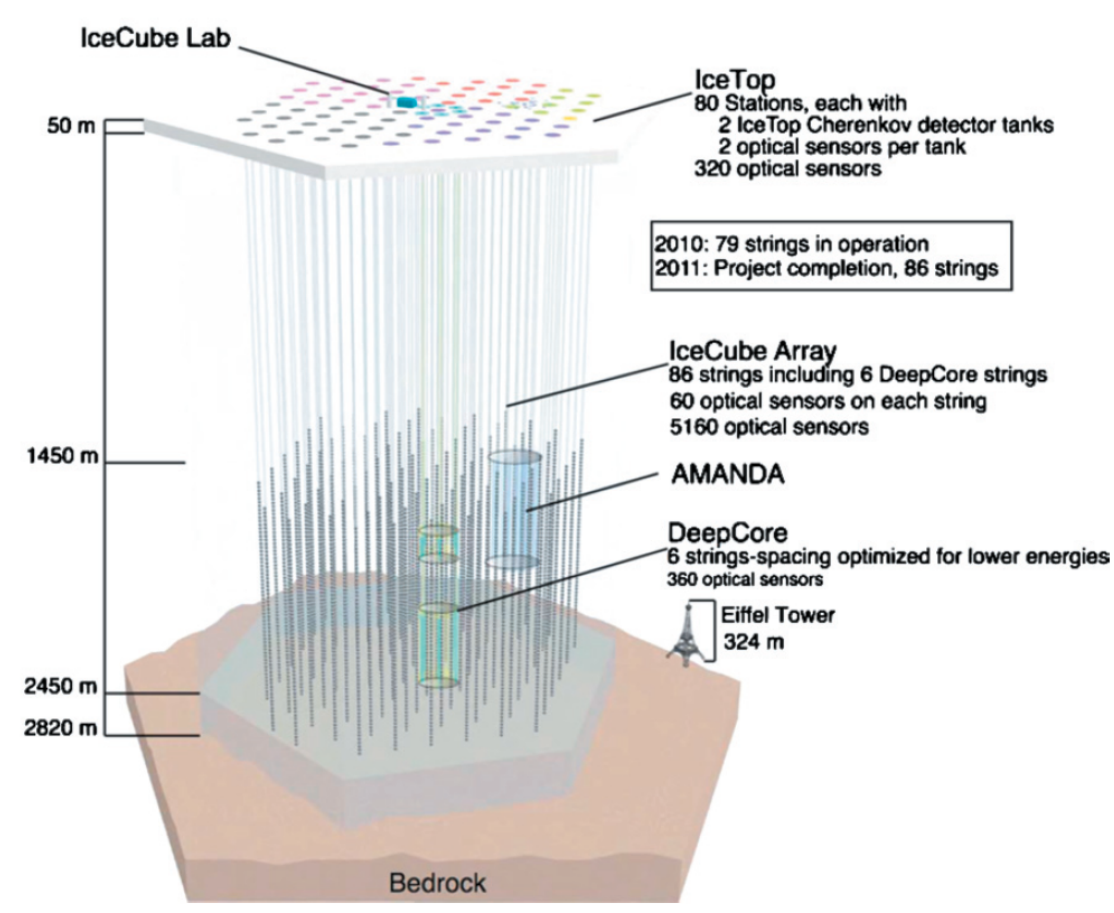


Figure 1. A cross section of the IceCube Neutrino Observatory, detailing depth, breadth, and the various components and experiments of the assemblage. Source: IceCube Collaboration.

The IceCube Collaboration comprises 45 scientific institutions from 12 countries and required an estimated 279 million US dollars to construct (IceCube Website). IceCube is comprised of 86 strings spaced 125 metres apart, each with 60 photoelectric sensors called Photomultiplier Tubes (PMT's) for a total of 5,160 Digital Optical Modules (DOMs). The PMT's are sensitive to Cherenkov radiation, a blue ultraviolet light that is emitted like a shockwave when particles decay from neutrino interactions and move faster than the speed of light can in the same icy medium. The DOM's analyze, amplify and digitize the information to send out of the ice to a lab on the surface, and to scientists around the world.

So far, one of the most significant contributions made by IceCube has been the detection of 28 high energy extragalactic neutrinos: those ranging in energy level from Terra Electron Volts (TeV) to Peta Electron Volts (PeV) (IceCube Collaboration, 2013; Aartsen et al., 2014). The acceleration of these high-energy neutrinos has remained a question for over 100 years. However, the most popular theory, consistent with IceCube's data, is that acceleration is generated in the shockfronts near black holes, gamma ray bursts, and supernovae (Aartsen et al., 2014). These rare extra-galactic neutrino events will eventually result in a new 'map' or 'view' of the astronomical sky.

As one of the largest and most sensitive technical instruments in the planet, IceCube is attuned to 25 orders of magnitude of energy levels (Halzen, 2015). IceCube's range of sensitivity is elegantly manifested in its capacity to sense extra-galactic neutrinos, but physicists at IceCube also study atmospheric neutrinos (those produced by cosmic rays interacting with the atmosphere), geo-neutrinos (those that decay from Earthly metals and may soon lead to a tomography, or scanning, of the whole Earth) and relativistic magnetic monopoles, or hypothetical particles that are predicted by quantum mechanics (but as yet unrecorded). Since some neutrinos pass through the entire planet to decay in the 
South Pole ice and thus be detected, the IceCube apparatus requires the material collaboration of the whole Earth, rendering the planet itself part of the sensing apparatus, and throwing into question the very definition of detection. Such highly distributed and unbounded modes of technical sensing challenge the use of fixed scales or "spheres" as frames of analysis. This challenge is particularly explicit in the performance of the neutrino cascade.

Cascading relations

The entity in question may be small, but its consequences may be quite profound. (Barad, 2007: 110)

In considering the intra-actions of matter-energy expressed in the IceCube apparatus, and by witnessing the cascades of subatomic particles caused by extra-galactic messengers, which spatial concepts become relevant? One spatial concept that emerged from our research into the IceCube Neutrino Observatory is the hadronic shower, or cascade. As we will describe further, cascades are fundamentally intra-active occasions that enfold information on neutrino particles, their energy levels and the cosmological objects from which they travel. In other words, cascades are spectacular examples of intra-action spanning the observable universe. They also demonstrate the value of intra-action for thinking and working beyond scalar regimes. The implications of neutrino cascades are far-reaching, especially as they provide information on neutrino 'mass hierarchies' that many physicists argue hold the key to understanding the formation of matter and anti-matter in the early universe. As we will show, they also unsettle assumptions behind the notion of a planetary technosphere.

Following scholars in geography and political ecology like Marston et al. (2005) and Swyngedouw (2004), we find that the presupposition of hierarchical scale - usually allied with a relatively small number of 'grains' (such as the body, neighborhood, urban, regional, national and global) 
- creates difficulties in thinking relations that exist outside, within, or intra- to, these terms. As Marston et al. (2005) assert, '... hierarchical scale is a classic case of form determining content, whereby objects, events, and processes come pre-sorted, ready to be inserted into the scalar apparatus at hand' (emphasis in original; Marston et al., 2005: 422). In other words, 'solving for scale' closes down the potential for unusual or surprising outputs and insights. Moreover, an analysis that relies on scale flirts with 'methodological perspectivalism, a God's Eye view' (ibid), which requires significant conceptual acrobatics to maintain. This is precisely the kind of "knowing at a distance" that is intrinsic to the technosphere concept as it has been developed thus far; it is the same gesture that Barad's agential realism questions and complicates.

Given its scale, degree of sensitivity and embeddedness in Antarctic Ice, the IceCube Neutrino Observatory can be described as an intra-active achievement of human practices, techno-science and Earthly matter. IceCube therefore invites us to think the technosphere otherwise, in terms that do not rely on scalar analysis, and that spring from the interstices of material and technical compositions. The highly dispersed agencies of matter, scientific theory, and technologies of sensing are beautifully manifested in the detection of spectral neutrino cascades generated by, "secondary charged particles produced in neutrino-matter interactions" (Taboada, 2014: 72).

The neutrino cascade is a "waterfall of causality in which one event triggers and affects the next" (italics ours; Fulton cited in Rooney, 2004: 176). While each cascade event occurs on a subatomic dimension, and is sensed via Cherenkov radiation, a cascade also transmits the direction, energy level, and location of the neutrino's astrophysical origin. In the case of high energy neutrinos, the origin might be thousands of light years away. In this way, subatomic and cosmological entities and forces are intimately entangled in the performance of a cascade. They cannot be separated into distinct or nested spheres. As they play across the IceCube apparatus, cascades might span the length of multiple football fields. These qualities lead us to understand the cascade as the expression of entanglement in the interstitial, liminal spaces between subatomic particles, an icy volume, black holes, digital sensors and 
ecologies of scientific practice. Sending shock waves across such assemblages, cascade phenomena collapse static, singular notions of scale(s) and amplify human, more-than-human as well as technical agencies.

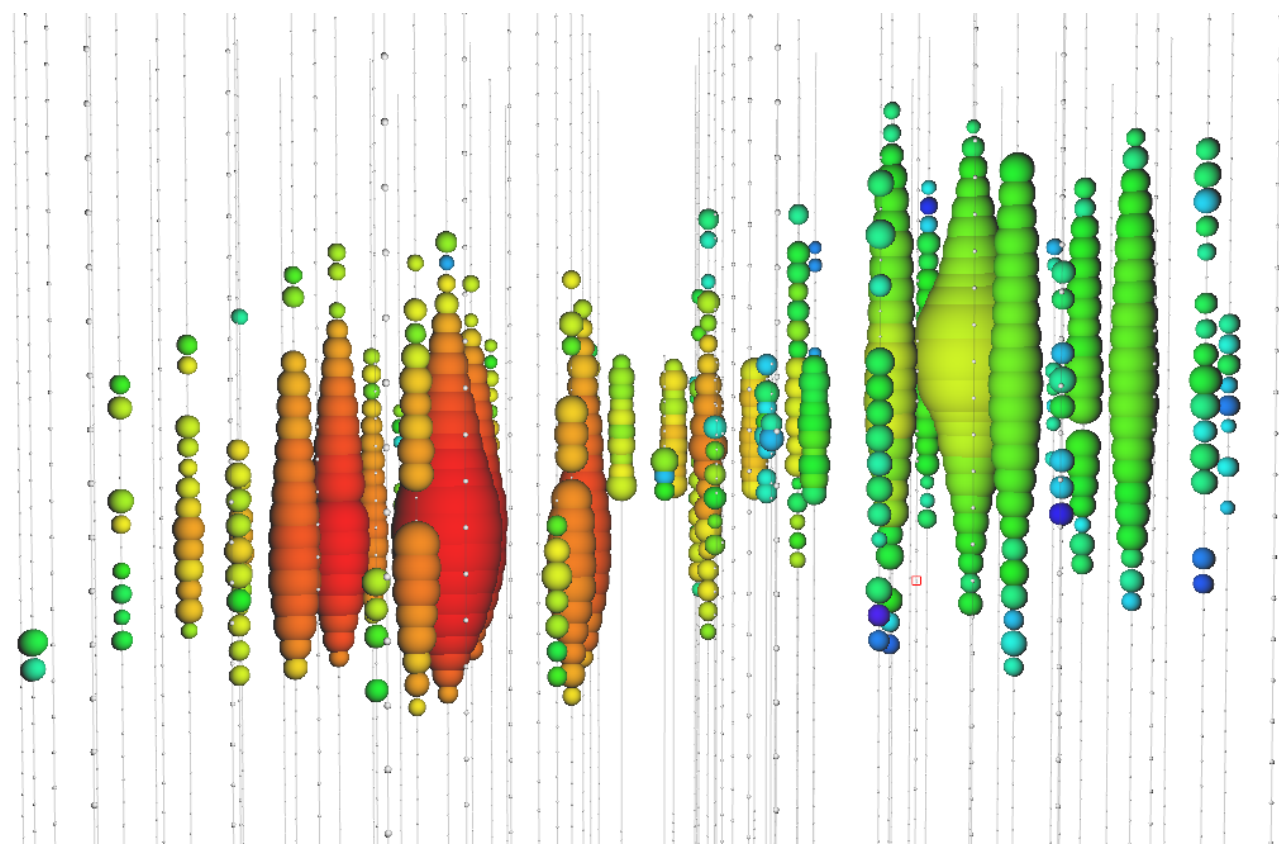

Figure 2. A “double bang” hadronic cascade. Source: IceCube collaboration.

Perhaps most strikingly, according to Dr. Halzen and the IceCube Collaboration, the qualities of neutrino cascade-measurements provide experimental evidence that the Standard Model of Particle Physics (SM) is flawed (Halzen, interview with authors, 2015). A new, 'exotic' model is therefore glimpsed as necessary to the process of 'meeting the Universe halfway' (Fulton cited in Barad, 2007: 397). It is not hyperbole to state that adjusting or revising the Standard Model to account for the insights of these experiments could embody a paradigm shift in proportion to that of the Copernican revolution. Such a shift has interesting consequences for the expression of our planet's technosphere insofar as it would shift access to the 'quality energy' that supports it. And it is precisely our ghost-like neutrino collaborators, their cascading intra-actions, and the entities constructed to detect them that are announcing 
new ways of thinking, doing, and engaging with dark and 'exotic' matters, energies and their compositions.

\section{Escaping the Coarse Grain}

As we have shown, there are several ways that static notions of scale limit the questions we can ask of technological infrastructures. As we will elaborate further in the paper's conclusion, practices of scaling and distancing are symptomatic of forms of knowledge production of the Earth System, and might be carefully and critically supplemented. Some of these limitations to research questions are indicated in the way the technosphere has heretofore been articulated. In a recent paper, Haff (2014b) proposes the technosphere is a geologic phenomenon by considering the 'commonalities' and 'contrasts' between it and other major planetary spheres like the hydrosphere, biosphere and lithosphere. The technosphere is compared to its spherical relatives using data on terawatts of energy absorbed, appropriation of resources or quantity of waste produced. The contribution to this special issue by Zalasiewicz et al. (2016) takes a similar approach by 'weighing' the technosphere. While such measurement-based studies are intriguing, they also collapse myriad questions of the complex dynamics between the technosphere and other agencies of our planet into two main modes (either commonalities or contrasts) and into assertions of relative magnitude. In this literature, relations between humans and the technosphere are often articulated as 'small-scale' and 'large-scale' components: as Haff (2014) writes, large-scale dynamics of a system appear 'spontaneously' and define, 'an environment within which small system components must operate' (Haff, 2014: 302). However, a better question might be: what is lost in the gesture of 'coarse graining' the relations between humans, non-humans, and technical infrastructures using categories like large and small?

Inspired by Barad's onto-epistemology, and taking cues from our engagement with the IceCube Neutrino Observatory, we might invent questions that do not establish scalar categories for worldly agencies. These questions would not compare and contrast, count or weigh, as much as they would seek 
to understand the dynamics of sites in which matter, skilled creatures, institutions and technologies exert pressure on each other in mutual co-evolution. In our research, for instance, we explored to what extent a single subatomic particle influences material, technical and scientific infrastructures. We found that a neutrino, detected as a cascade in the ice of Antarctica, sends ripples and shock-waves through sensing devices, computational infrastructures, scientific collaborations, theories and imaginaries of the universe. While we recognize the usefulness of the 'coarse-graining' analysis favored by Haff (2014) and others in complex systems studies, we feel such a scale-based approach does not fully account for technologies' embeddedness in restless matters, theories, practices and experiments of the Anthropocene. These are entities and processes that cannot be isolated nor abstracted into grains, coarse or otherwise. (At which 'grain' would one find the neutrino?) A more careful consideration of notions of scale is generated by sensitivity to intra-action. However it is necessary to emphasize that, like concepts of scale, intra-action cannot be taken for granted: it is emergent from the world, rather than an abstract lens we can use to view and know the world. In this paper, the particular form and evidence of intra-action, as well as the "spatial concept" performed in IceCube, is the neutrino cascade. Intra-action and spatial concepts might be linked and performed in myriad other situated styles and dimensions. In light of these arguments and insights, we can now ask: are there ways to recuperate the technosphere concept? Can the gestures of 'graining' and distancing underpinning the technosphere concept as it is conceived thus far be critically reassessed and complemented? In other words: are there cascades in the technosphere?

\section{The neutrino sky}

To address these questions, we return to the IceCube Neutrino Observatory to elaborate a few ways in which the technosphere concept might be brought into conversation with post-humanist thought. Our aim is not to propose an alternative to the technosphere concept, as such a project would outrun the scope of this commentary paper. However, we will put forward ideas for further critique and discussion that emerge from our engagement with IceCube. First, the challenge with which we began this paper - of 
becoming adequate, in both thought and action, to the 'escaped velocities' of technological infrastructures - can best be achieved by a willingness to invent-with, and be affected-by, the expressiveness of sites and situated voices. As IceCube renders spectacularly evident, the project of becoming-sensitive to the traces of 'cosmic messengers' troubles all kinds of boundaries and boundary-making discourses that place technology on a different plane from ice sheets, rocks, humans or neutrinos. Perhaps, if the technosphere were instead conceived of as multiple, as myriad spheres in constant formation, the specificity and expressiveness of sites could be recuperated. However, we are skeptical that any project founded on the abstraction of a 'sphere' can truly capture the qualities of technical agencies (or any form of agency for that matter).

Second, our engagement with neutrinos via the IceCube Observatory points to the possibility that the assumptions underlying the technosphere's search for 'quality energy' might bear revision. Since the technosphere (as described by Haff) ultimately seeks 'quality energy', and the Standard Model (SM) is the 'rule book' for how we understand energy and matter, a revision of SM could lay the foundation for the discovery of much more efficient energy sources. If revising SM leads us one step closer to an identification of dark matter or energy, our planet's material-energetic and technical constitution might be radically shifted. Just as we must become adequate to the technical conditions with which we dwell, technology itself will undoubtedly be altered and challenged by a new horizon of scientific description of the universe. Interestingly, and perhaps paradoxically, the phantom-like neutrino is one of the 'messengers' of these earthly-cosmic horizons. But perhaps this is not paradoxical at all: the gesture of reaching for the imperceptible, of sensing at, or across, the limit, generates methods and spaces of insight that are precluded by gestures of bracketing, framing or graining, however sophisticated the resolution may be. We are aware that every scientific practice, even that of neutrino sensing, involves setting limitations that allow research questions to be posed. However, the project of crafting a description of technical infrastructure that is sensitive to scale and agency in the Anthropocene is an ambitious and demanding task: one that does not belong to scientists alone. And it is a task that should welcome those adventures in research and detection that extend bodies, matters and devices to the edges of their sensual 
worlds, inviting unusual insights and phenomena into the space of research. The boundary-making practices implicit in the technosphere concept require crucial adjustments to account for myriad phenomena operating across spaces and scales, especially evidenced here in the neutrino cascade.

Thirdly, we want to pose an open question about the kinds of knowledge production supporting the technosphere concept as it resonates within Earth System Science more generally. As Clive Hamilton (2016) has articulated in this journal, Earth System Science marks a paradigm shift in scientific attention to our planet. The Earth System is a new object: 'a single, dynamic, integrated system' (Hamilton, 2016: 94). In its espousal of a systems-level 'rupture' in planetary history, this conception of the Earth System resonates with the claims of Haff's technosphere concept. Both concepts, however, are predicated on ways of knowing that pre-suppose an external viewpoint: a gesture of stepping outside of the system (or the technosphere) in order to observe the system itself. Once again, Barad's words offer a radically different stance: 'Knowing does not come from standing at a distance and representing, but from a direct material engagement with the world' (Barad, 2007: 49). The principles of agential realism and intraaction hold firmly that a scientific description of the Earth System or technosphere changes the very assemblage it seeks to describe, and is itself mutually altered. An interesting alternative proposal that avoids a distancing, representational stance is the critical zone: a concept that has also emerged from the earth sciences, especially from research on water catchments, to describe a trans-scalar and transdisciplinary focus on Earth's "fragile envelope" (Brantley et al., 2007; Latour, 2014). The CZ is merely one proposition; there are myriad other research frameworks that enable a Baradian situation in which scientists and practitioners are always already within the zone of study, rather than outside it.

In this vein, we must also summon Benjamin Bratton's The Stack, an 'accidental megastructure' that offers valuable insights into the geo-political implications of 'planetary scale computation' (Bratton, 2016). Bratton describes six trans-scalar 'layers' that mutually reinforce each other. Beginning with the Earth Layer, where 'quality energy' is extracted and processed, and moving to Cloud, City, Address, Interface, and User, Bratton addresses the political geographies of, 'the planet under an absolutist regime of algorithmic capital' (Bratton, 2016: 72). Crucially, Bratton describes this project as a design, both 
intentional and unintentional. The Stack is a model 'that both does and does not exist' (Bratton, 2014: np). Moreover, he asserts, "[W]e become what we are by making that which in turn makes us" (Bratton, 2016: xvii). Such an account is compelling not only for its nuanced articulation of categories that are porous, changing and reassembling, but also since The Stack is continuously reconfigured (even potentially dissolved) by the agencies, politics and chemical elements that it renders increasingly legible.

In resonance with this thinking, our in-depth engagement with one technical assemblage - the IceCube Neutrino Observatory - suggests to us that any concept of Earth's technical capacity requires modes of analysis that transcend scale-based comparison and accommodate the redistribution of agency established in agential realism and posthumanism. Echoing Barad, we find that profound ripples in technological infrastructures are triggered by the 'very small,' even the subatomic. Consequently, as scholars like Bratton also emphasize, assertions of boundedness between the spheres of the biological and the technical, or the Earth and its technosphere, belie only the nascent state of our knowledge of such assemblages. Critical endeavors to understand the technical capacities of our planet require that we better attune to the intra-actions between the breathtakingly complex ecologies of technology, matter, and energy, and listen to the various 'cosmic messengers' along the way. 


\section{References}

Barad K (2007) Meeting the universe halfway: Quantum physics and the entanglement of matter and meaning. London: Duke University Press.

Barad K (2014) Posthumanist performativity: Toward an understanding of how matter comes to matter. Signs 40(1).

Bennet J (2010) Vibrant Matter: Towards a political ecology of things. London: Duke University Press. Braidotti R (2013) The Posthuman. UK: Polity Press.

Brantley, S. L., Goldhaber, M. B., \& Ragnarsdottir, K. V. (2007) Crossing disciplines and scales to understand the critical zone. Elements, 3(5): 307-314.

Bratton B (2014) The Black Stack. E-Flux Journal, 53.

Bratton B (2016) The stack: On software and sovereignty. Cambridge, MA: MIT Press.

Casey S and Ellis R (2015) Dark Matters Project. Presentation at RGS-IBG conference, Exeter University, 2-4 September 2015.

Gaisser TK, Halzen F, and Stanev T (1995) Particle astrophysics with high energy neutrinos. Physics Reports 258(3): 173-236.

Haff P (2014a) Humans and technology in the Anthropocene: Six rules. The Anthropocene Review 1(2): 126-136.

Haff P (2014b) Technology as a geological phenomenon: implications for human well-being. in Waters, C. N., Zalasiewicz, J. A., Williams, M., Ellis, M. A. \& Snelling, A. M. (eds) A Stratigraphical Basis for the Anthropocene. Geological Society, London: Special Publications, 301-309.

Haff P (2015) Quantum mechanics as one of the key triggers of the technosphere. Public presentation in 'Triggers: introducing the Technosphere' 100 Years of Now: The Opening. Haus der Kulturen der Welt, Berlin, September 30 - October 4. https://www.hkw.de/en/app/mediathek/video/43982

Halzen F (1998) The AMANDA neutrino telescope. New Astronomy Reviews 42(3): 289-299. 
Halzen F (2015) Interview with authors. August 7, 2015.

Halzen F and Klein SR (2010) IceCube: An instrument for neutrino astronomy. Review of Scientific Instruments 81(8): 081101.

Haraway D (1997) Modest_Witness@Second_Millenium.FemaleMan@_Meets_OncoMouse ${ }^{\mathrm{TM}}$. Feminism and Technoscience. New York: Routledge.

Holmes D R, \& Marcus G E (2008) Collaboration today and the re-imagination of the classic scene of fieldwork encounter. Collaborative anthropologies 1(1): 81-101.

Latour B (2014) Some advantages of the notion of "Critical Zone" for geopolitics. Procedia Earth and Planetary Science, 10, 3-6.

Marston SA, Jones JP, and Woodward K (2005) Human geography without scale. Transactions of the Institute of British Geographers 30(4): 416-432.

Massumi B (2002) A shock to thought: Expression after Deleuze and Guattari. New York: Routledge.

Nancy JL (2000) Being singular plural. Trans. Richardson, R. \& O’Byrne A. Stanford: Stanford University Press.

Nancy JL (2008) Corpus. Trans. Rand R. New York: Fordham University Press.

Rooney K (2004) Review of Cascade Experiment: Selected Poems. Harvard Review (27): 176-178. Retrieved from http://www.jstor.org/stable/27568963

Swyngedouw E (2004) Scaled geographies: Nature, place, and the politics of scale. Scale and geographic inquiry: Nature, society, and method. Oxford: Blackwell, 129-153.

Szerszynski B (2014) The Onomatophore of the Anthropocene. Presentation at EmbraceConfusion Project Space, Berlin, August 20, 2014.

Taboada I (2013) Neutrino astrophysics with IceCube. Neutrino Instruments and Methods in Physics Research A A (742): 71-76. 